\title{
Effects of exercise training on gingival oxidative stress in obese rats
}

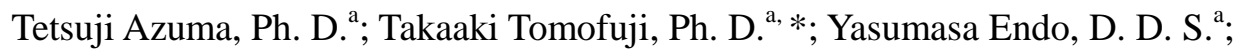

Naofumi Tamaki, Ph. D. ${ }^{a}$; Daisuke Ekuni, Ph. D. . $;$ Koichiro Irie, D. D. S. ${ }^{a}$;

Kenta Kasuyama, D. D. S. ${ }^{a}$; Tomo Kato ${ }^{a}$; and Manabu Morita, Ph. D. ${ }^{a}$

${ }^{\text {a }}$ Department of Preventive Dentistry, Okayama University Graduate School of

Medicine, Dentistry and Pharmaceutical Sciences, 2-5-1 Shikata-cho, Okayama 700-8558, Japan

* Corresponding author: Takaaki Tomofuji, Department of Preventive Dentistry,

Okayama University Graduate School of Medicine, Dentistry and Pharmaceutical

Sciences, 2-5-1 Shikata-cho, Kita-ku, Okayama 700-8558, Japan;

Tel: +81 86235 6712; Fax: +81 86235 6714; E-mail: tomofu@md.okayama-u.ac.jp

Running title: Exercise, obesity and periodontal health

Key words: obesity; exercise training; periodontal diseases; oxidative stress 
List of abbreviations: CRP, C-reactive protein; GSH, reduced-form glutathione; GSSG, oxidized-form glutathione; 8-OHdG, 8-hydroxydeoxyguanosine; ROM, reactive oxidative metabolites; ROS, reactive oxygen species; TRAP, tartrate-resistant acid phosphatase 


\begin{abstract}
Objective: The purpose of the present study was to investigate the effects of exercise training on serum reactive oxygen species (ROS) level and gingival oxidative stress in obese rats fed a high-fat diet.
\end{abstract}

Design: Rats were divided into three groups ( $\mathrm{n}=14 /$ group): one control group (fed a regular diet) and two experimental groups (fed a high-fat diet with and without exercise training [treadmill: 5 days/week]). The rats were sacrificed at 4 or 8 weeks. The level of serum reactive oxidative metabolites (ROM) was measured as an indicator of circulating ROS. The level of 8-hydroxydeoxyguanosine (8-OHdG) and reduced-form glutathione (GSH)/oxidized-form glutathione (GSSG) ratio were determined to evaluate gingival oxidative stress.

Results: The obese rats fed a high-fat diet without exercise training showed higher serum ROM levels [Carratelli Units (CARR U)] (mean \pm SD; $413 \pm 64$ ) than the control $(333 \pm \mathbf{1 2})$ at 4 weeks $(p=\mathbf{0 . 0 2 3})$. Such a condition resulted in higher 8 -OHdG levels (ng/mg mtDNA) $(\mathbf{0 . 9 7} \pm \mathbf{0 . 1 8})(p<0.05)$ and a lower GSH/GSSG ratio $(\mathbf{1 7 . 0} \pm \mathbf{3 . 1})$ $(p<0.05)$ in gingival tissues, compared to the control $(0.55 \pm 0.13$ for 8 -OHdG and 23.6 \pm 5.8 for GSH/GSSG ratio) at 8 weeks. In addition, the obese rats fed a high-fat diet with exercise training showed lower serum ROM $(623 \pm 103)(p<0.001)$ and gingival 
8-OHdG levels $(0.69 \pm \mathbf{0 . 1 7})(p=\mathbf{0 . 0 1 2})$ than those without exercise training $(1105 \pm 95$ for ROM and $\mathbf{0 . 5 5} \pm \mathbf{0 . 1 3}$ for $8-\mathrm{OHdG})$ at 8 weeks.

Conclusions: Obesity prevention by exercise training may effectively suppress gingival oxidative stress by decreasing serum ROS in rats. 


\section{Introduction}

Obesity is a chronic metabolic problem with important public health concerns. There is increasing evidence linking obesity to periodontal disease. Cross-sectional studies have shown a positive relationship between body mass index and periodontal diseases. ${ }^{1-3}$ Animal studies also revealed that the gingiva in obese rats exhibited higher densities of polymorphonuclear leukocytes than that in normal rats. ${ }^{4,5}$ Thus, obesity may be a risk factor for periodontal diseases.

Adipose tissue secretes several bioactive substances, including reactive oxygen species (ROS). ${ }^{6}$ Studies have shown that obesity is associated with a state of systemic increase in ROS. $^{7,8}$ ROS are products of normal cellular metabolism. However, overproduction of ROS may disturb the tissue oxidative/anti-oxidative balance, which causes oxidative stress by damaging DNA, lipids and protein.? Recent clinical studies suggested that increased oxidative stress and/or decreased anti-oxidative status are involved in the progression of periodontal diseases. ${ }^{10-12}$ In a rat model, gingival oxidative stress was associated with periodontal inflammation ${ }^{4}$ and osteoclast differentiation on alveolar bone surface. ${ }^{13}$ Therefore, a systemic increase in ROS following obesity may lead to progression of periodontal inflammation and osteoclast differentiation through gingival oxidative stress. 
Obesity is also associated with systemic inflammation, which is characterized by increased levels of circulating C-reactive protein (CRP). ${ }^{14}$ A systematic review shows strong evidence that plasma CRP in periodontitis patients is elevated compared with control subjects. ${ }^{15}$ CRP is known to induce oxidative stress. ${ }^{16,17}$ The mechanism by which CRP affects periodontal tissues is unclear; however, elevated blood levels of CRP in obesity may also cause gingival oxidative stress.

Regular exercise offers protection against chronic conditions such as obesity. ${ }^{18}$ Protective mechanisms include regulation of body weight and reduction of inflammation in adipose tissue. ${ }^{19-21}$ Studies suggest that the reduction of body fat causes a decrease in the formation of oxidants ${ }^{21-23}$ and systemic inflammation. ${ }^{24}$ Therefore, it is possible that prevention of fat accumulation by exercise training inhibits overproduction of ROS and reduces inflammation in the adipose tissue. Such conditions would reduce levels of circulating ROS and systemic inflammation in obesity.

In the present work, we hypothesized that obesity prevention by exercise training might prevent gingival oxidative stress by suppressing the systemic increase of ROS and CRP. 8-hydroxydeoxyguanosine $(8-\mathrm{OHdG})$ is the DNA fragment. It is formed when guanine in DNA receives oxidative stress. ${ }^{25}$ It is also known that glutathione is a ubiquitous tripeptide that plays a central role in cellular anti-oxidant defense. 
Oxidized-form glutathione (GSSG) accumulates inside the cells, while reduced-form glutathione (GSH) is decreased by oxidative stress. ${ }^{26}$ Therefore, the purpose of this study was to investigate the preventive effects of exercise training on serum ROS level, serum CRP level, gingival 8-OHdG level and gingival GSH/GSSG ratio in obese rats fed a high-fat diet. Serum level of reactive oxidative metabolites (ROM) was measured as an indicator of circulating ROS. ${ }^{12}$ The main components of ROM are hydroperoxides (intermediate oxidative products of lipids, peptides and aminoacids), and analysis of these can help to determine the total oxidative status in serum. Furthermore, histopathological evaluation, including inflammatory cell infiltrate and osteoclast differentiation, was performed to confirm whether obesity prevention by exercise training could prevent periodontal disease. 


\section{Materials and Methods}

\subsection{Animals}

Forty-two male Wistar rats ( 8 weeks old) were used in this study. The animals were housed under standard conditions and the room was maintained on a cycle of 12-hour light and 12-hour darkness and at a temperature of $23^{\circ} \mathrm{C}$ to $25^{\circ} \mathrm{C}$. The experimental protocol was approved by the Animal Research Control Committee of Okayama University Graduate School of Medicine, Dentistry and Pharmaceutical Sciences.

\subsection{Experimental design}

Animals were randomly assigned to either a high-fat $(n=28)$ or a regular diet protocol $(n=14)$. The high-fat diet consisted of $32.6 \%$ lipid, $43.8 \%$ carbohydrate, and $23.5 \%$ protein (kcal) (F2HFD1, Oriental Yeast Co., Osaka, Japan). ${ }^{27}$ The regular diet $(12.5 \%$ lipid, $63.2 \%$ carbohydrate, and $24.3 \%$ protein; kcal) consisted of usual pellet rat chow. Animals fed the high-fat diet were divided into two groups. One group of rats $(n=14)$ remained sedentary for the 4- or 8-week period (HF-Sed, $n=7$ each). The other group of rats $(n=14)$ was exercise trained for 4 or 8 weeks (HF-Tr, $n=7$ each). Animals fed the regular diet remained sedentary for the 4 or 8 weeks (RD-Sed, $n=7$ each). Exercise training consisted of continuous running on a motor-driven rodent treadmill (Agawa 
Machine Co., Shimane, Japan) 5 times a week. Exercise intensity was progressively increased from 15 minutes/day at $15 \mathrm{~m} /$ minute, $0 \%$ slope, up to 60 minutes/day at 26 $\mathrm{m} /$ minute, $10 \%$ slope, in the last 4 weeks of the program. ${ }^{28}$ The condition of the last 4 weeks corresponded to a moderate intensity of $\sim 65 \%$ of maximum oxygen consumption. $^{29}$

\subsection{Histological analyses}

After the animals were sacrificed under general anesthesia, the maxillary molar regions (tooth and periodontal tissues) were resected from each rat and immediately fixed with 4\% paraformaldehyde in $0.1 \mathrm{~mol} / \mathrm{L}$ phosphate buffer $(\mathrm{pH} 7.4)$ for 1 day. The entire abdominal white adipose tissue (visceral fat) was also dissected and weighed. The tooth and periodontal tissues were decalcified with $10 \%$ tetrasodium-ethylenediaminetetraacetic acid aqueous solution ( $\mathrm{pH} 7.4$ ) for 14 days at $4^{\circ} \mathrm{C}$. Paraformaldehyde-fixed periodontal and adipose tissue samples were embedded in paraffin and stained with hematoxylin and eosin. In periodontal tissue sections, tartrate-resistant acid phosphatase (TRAP) activity was also detected by the azo dye method to identify osteoclasts (Fig. 1). ${ }^{13}$ Histological changes were evaluated using a microscope (BX 51-54, Olympus Co., Tokyo, Japan) by one examiner, who was blinded 
to the treatment assignment. In the periodontal tissue, the polymorphonuclear leukocytes per standard area $(0.05 \mathrm{~mm} \times 0.1 \mathrm{~mm})$ were counted in the connective tissues subjacent to the junctional epithelium. ${ }^{4}$ The distance between the cemento-enamel junction and alveolar bone crest was also measured to evaluate the level of alveolar bone resorption. ${ }^{4}$ TRAP-positive osteoclasts occurring along the edge of the alveolar bone surface were counted and reported as number $/ \mathrm{mm} .{ }^{13}$

\subsection{Measurement of the gingival 8-OHdG level}

Mitochondrial DNA was isolated from rat gingiva using a DNA extractor kit (Wako Pure Chemical Industries, Osaka, Japan). Level of $8-\mathrm{OHdG}$ in the isolated mitochondrial DNA was analyzed using an ELISA kit (Japan Institute for the Control of Aging, Shizuoka, Japan). ${ }^{30}$

2.5. Measurements of the gingival glutathione level

The levels of total glutathione (GSH + GSSG level) and GSSG were determined with colorimetric assay kits (Dojindo Laboratories, Tokyo, Japan). ${ }^{30}$ GSSG was determined after blocking GSH with 2-vinylpyridine and the GSH/GSSG ratio in each sample was calculated. 


\subsection{Measurements of circulating ROM and CRP}

Blood samples were collected after 8 weeks directly from the heart of 24-hour-fasted animals. Blood was allowed to clot at room temperature, and serum was separated by centrifugation at $1,500 \times g$ for 15 minutes. An $N, N$-diethylparaphenylendiamine ROM test was performed immediately using the free radical elective evaluator (Diacron, Grosseto, Italy) on the same day of blood sampling. ${ }^{12}$ This measurement evaluates total hydroperoxides, which are generated by the action of ROS on lipids, peptides and aminoacids and their level reflects the total oxidative burden. The measurement unit was expressed as conventional units (Carratelli Units, where 1 CARR U corresponds to $0.08 \mathrm{mg} / \mathrm{dL} \mathrm{H}_{2} \mathrm{O}_{2}$ ). In addition, the remaining serum aliquots were stored at $-80{ }^{\circ} \mathrm{C}$, and serum levels of C-reactive protein (CRP) were later quantified by a highly sensitive ELISA (Life Diagnostics, Inc., West Chester, UK) after thawing all serum samples. ${ }^{5}$

\subsection{Statistical Analysis}

Parameters were analyzed statistically using one-way ANOVA followed by Tukey's method (for comparisons among the RD-Sed, HF-Sed and HF-Tr groups at 4 or 8 weeks) and $t$-test (for comparison between 4 and 8 weeks within the RD-Sed, 
HF-Sed or HF-Tr groups). All analyses were performed using a software program (SPSS 15.0 J for Windows, SPSS Japan, Tokyo, Japan). $P<0.05$ was considered to be statistically significant. 


\section{Results}

3.1. Food consumption, body weight, and adipose tissue weight

There were no significant differences in food consumption among the three groups during the experimental period. Because the foot of a rat in the HF-Tr group was injured during exercise training, the data of this animal were removed from our results.

At baseline, no significant differences in body weight were found among the three groups ( $p>0.05)$ (Table 1). The rats in the HF-Sed group had greater body weight $(p<$ $0.05)$, visceral fat pad weight $(p<0.05)$, and subcutaneous fat pad weight $(p<0.05)$ than those in the RD-Sed group at 4 and 8 weeks. The rats in the HF-Tr group also had greater visceral fat pad weight $(p<0.05)$ and subcutaneous fat pad weight $(p<0.05)$ than those in the RD-Sed group at 4 weeks. However, the rats in the HF-Tr group had lower visceral fat pad weight $(p<0.05)$ and subcutaneous fat pad weight $(p<0.05)$ than those in the RD-Sed and HF-Sed groups at 8 weeks. In addition, in the RD-Sed and HF-Sed groups, body weight, visceral fat pad weight and subcutaneous fat pad weight at 8 weeks increased compared to those at 4 weeks $(p<0.05)$. In the HF-Tr group, subcutaneous fat pad weight at 8 weeks decreased compared to that at 4 weeks $(p<$ $0.05)$, while body weight at 8 weeks increased compared to that at 4 weeks $(p<0.05)$. 


\subsection{Histological evaluation}

At 4 weeks, there were no significant differences in polymorphonuclear leukocyte density $(p>0.05)$, distance between the cemento-enamel junction and alveolar bone crest $(p>0.05)$, and number of TRAP-positive osteoclasts $(p>0.05)$ among the three groups (Table 2). At 8 weeks, the polymorphonuclear leukocyte density and number of TRAP-positive osteoclasts in the HF-Sed group were higher than those in the RD-Sed group $(p<0.05)$ (Fig. 1). The numbers of polymorphonuclear leukocytes and TRAP-positive osteoclasts in the HF-Tr group were lower than those in the HF-Sed group at 8 weeks $(p<0.05)$. No significant differences in the distance between the cemento-enamel junction and alveolar bone crest were observed among the three groups at 8 weeks $(p>0.05)$. Furthermore, in the HF-Sed group, distance between the cemento-enamel junction and alveolar bone crest and the number of TRAP-positive osteoclasts at 8 weeks increased compared to those at 4 weeks $(p<0.05)$. In the HF-Tr group, the polymorphonuclear leukocytes density at 8 weeks decreased compared to that at 4 weeks $(p<0.05)$.

\subsection{Gingival 8-OHdG level and GSH/GSSG ratio}

At 4 weeks, the gingival 8-OHdG level of the HF-Sed and HF-Tr groups was $115 \%$ and 
$109 \%$ higher compared with that of the RD-Sed group $(p<0.05)$ (Table 3). The gingival 8-OHdG level of the HF-Sed group was $76 \%$ higher compared with that of the RD-Sed group at 8 weeks $(p<0.05)$. The gingival $8-\mathrm{OHdG}$ level of the HF-Tr group was $29 \%$ lower compared with that of the HF-Sed group at 8 weeks $(p<0.05)$. No significant difference in gingival 8-OHdG level between 4 and 8 weeks was found in any group ( $p>$ 0.05). In addition, the gingival GSH/GSSG ratio of the HF-Sed group was $28 \%$ lower compared with that of the RD-Sed group at 8 weeks $(p<0.05)$. This value decreased compared to that at 4 weeks $(29 \%)(p<0.05)$. There was no significant difference in gingival GSH/GSSG ratio between the RD-Sed and HF-Tr groups at 8 weeks $(p>0.05)$.

\subsection{Plasma ROM and CRP levels}

At 4 weeks, the serum levels of ROM and CRP in the HF-Sed group were higher than in the RD-Sed group ( $p<0.05$ ) (Table 4). The serum level of ROM in the HF-Tr group was also higher than in the RD-Sed group at 4 weeks $(p<0.05)$. At 8 weeks, the serum levels of ROM and CRP in the HF-Sed group were higher than in the RD-Sed group $(p<0.05)$.

On the other hand, the serum levels of ROM and CRP in the HF-Tr group were lower than in the HF-Sed group at 8 weeks $(p<0.05)$. Furthermore, in all groups, the serum ROM level at 8 weeks increased compared to that at 4 weeks $(p<0.05)$, while there was 
no significant difference in serum CRP level between 4 and 8 weeks $(p>0.05)$. 


\section{Discussion}

In the present study, the rats fed a high-fat diet showed greater body weight, fat accumulation and the serum ROM level than those fed the control diet at 4 weeks. Such conditions induced a high 8-OHdG level and a low GSH/GSSG ratio in gingival tissues. The polymorphonuclear leukocyte infiltration and number of TRAP-positive osteoclasts on the alveolar bone surface was greater than the control at 8 weeks. On the other hand, exercise training suppressed the effects of the high-fat diet on gingival 8-OHdG level, polymorphonuclear leukocyte infiltration and the formation of TRAP-positive osteoclasts at 8 weeks, thus suppressing fat accumulation and lowering serum ROM elevation. Exercise training can thus effectively suppress the effects of obesity on gingival oxidative stress and periodontal disease by decreasing circulating ROS.

Previous studies have shown that regular exercise markedly reduced oxidative stress (plasma malondialdehyde level) in obese, type 2 diabetic patients ${ }^{31}$ and improved blood level of oxidative stress-defense parameters in overweight and obese type 2 diabetics. ${ }^{32}$ These observations are consistent with the present results, and it is feasible that the suppression of obesity by exercise training might contribute to a decrease in circulating ROS. Moreover, in this study, the rats with exercise training showed a gingival 8-OHdG level lower than the group without exercise training at 8 weeks. These 
findings indicate that exercise training could improve local alterations in gingival redox balance as well as decrease circulating oxidative stress in obesity.

A clinical study revealed that fat accumulation is closely correlated to the systemic increase of ROS. ${ }^{33}$ In the present study, exercise training suppressed fat accumulation and lowered serum ROM level in the rats fed a high-fat diet. These findings indicate that the preventive effects of exercise training on fat accumulation resulted in suppression of serum ROS elevation. On the other hand, although the body weight and adipose tissue weight in the obese rats with exercise training were lower than those in the control rats, there were no significant differences in serum ROM level between these two groups at 8 weeks. Even if exercise training can reduce fat accumulation below the normal level, its suppressive effects on serum ROS level may have a limit.

We found that exercise training did not modulate the effects of a high-fat diet on fat pad weight at 4 weeks. Furthermore, the levels of serum ROM and gingival 8-OHdG did not differ in obese rats with and without exercise training at 4 weeks. It is reported that fat accumulation causes an increase in the formation of oxidants. ${ }^{21-23}$ In addition, it is known that the degree of gingival oxidative stress is associated with the blood ROS level. ${ }^{4}$ Therefore, because fat accumulation did not change, it is feasible that exercise training had little effect on gingival oxidative stress at 4 weeks. An exercise period 
above a certain level may be necessary to suppress gingival oxidative stress following obesity.

Recent animal studies have demonstrated that osteoclast differentiation is stimulated by oxidative stress. ${ }^{13,34}$ This evidence indicates that oxidative stress plays a crucial role in osteoclast differentiation. In this study, the obese rats with exercise training exhibited a lower number of TRAP-positive osteoclasts than those without exercise training at 8 weeks. Reduction of gingival oxidative stress by exercise training may result in inhibition of osteoclast differentiation induced by obesity. However, the distance between the cemento-enamel junction and alveolar bone crest did not change following obesity or exercise training during the experimental period. In our model, at 8 weeks, the degree of gingival oxidative stress induced by obesity might be enough to induce osteoclast differentiation, but not enough to influence alveolar bone resorption (e.g., alveolar bone height). On the other hand, it is also possible that alveolar bone resorption will be observed in a later evaluation. Thus, it may take more periods to observe alveolar bone resorption by obesity in this model.

At 4 weeks, obesity increased the gingival 8-OHdG level, but it did not affect the gingival GSH/GSSG ratio. Such conditions did not show any pathological changes in the periodontal tissue. On the other hand, at 8 weeks, obesity induced both a higher 
gingival 8-OHdG level and a lower gingival GSH/GSSG ratio than the control, resulting in osteoclast differentiation on alveolar bone. The combination of oxidative stress and anti-oxidant defense in gingival tissues might be responsible for inducing alveolar bone loss.

Studies have demonstrated that exercise training has an anti-inflammatory effect in obesity. ${ }^{35,36}$ In the present results, the plasma CRP level in obese rats with exercise training was lower than in those without exercise training. Therefore, it is possible that the decreases in not only blood ROS but also low-grade systemic inflammation had beneficial effects on gingival oxidative stress. In the future, additional assays for measuring pro-inflammatory cytokines in serum and gingival samples may be required to investigate whether exercise training induces any antioxidative or anti-inflammatory effects.

The present study had a few limitations. For instance, the present study did not include a non-obese group that received exercise training. Therefore, the actual effect of exercise training on the normal gingiva remains unclear. In addition, we did not examine alveolar bone density. It is possible that there were significant differences in alveolar bone density among the three groups, although the distance between the cemento-enamel junction and alveolar bone crest did not differ in our results. It would 
be necessary to investigate how obesity and exercise training affect alveolar bone density in the present rat model.

In conclusion, in the rat model, exercise training could inhibit obesity-induced gingival oxidative stress, periodontal inflammation, and osteoclast differentiation by decreasing the serum ROS and CRP levels. 


\section{Acknowledgements}

This study was supported by Grants-in-Aid for Scientific Research (21792150) from the

Ministry of Education, Culture, Sports, Science and Technology, Tokyo, Japan. 


\section{References}

1. Al-Zahrani MS, Bissada NF, Borawskit EA. Obesity and periodontal disease in young, middle-aged, and older adults. J Periodontol 2003; 74: 610-615.

2. Saito T, Shimazaki Y, Kiyohara Y, et al. Relationship between obesity, glucose tolerance, and periodontal disease in Japanese women: the Hisayama study. $J$ Periodontal Res 2005; 40: 346-353.

3. Wood N, Johnson RB, Streckfus CF. Comparison of body composition and periodontal disease using nutritional assessment techniques: Third National Health and Nutrition Examination Survey (NHANES III). J Clin Periodontol 2003; 30: 321-327.

4. Tomofuji T, Yamamoto T, Tamaki N, et al. Effects of obesity on gingival oxidative stress in a rat model. J Periodontol 2009; 80: 1324-1329.

5. Endo Y, Tomofuji T, Ekuni D, et al. Experimental periodontitis induces gene expression of pro-inflammatory cytokines in liver and white adipose tissues in obesity. J Periodontol 2010; 81: 520-526.

6. Matsuzawa-Nagata N, Takamura T, Ando H, et al. Increased oxidative stress precedes the onset of high-fat diet-induced insulin resistance and obesity. Metabolism 2008; 57: 1071-1077. 
7. Mohora M, Vîrgolici B, Paveliu F, Lixandru D, Muscurel C, Greabu M. Free radical activity in obese patients with type 2 diabetes mellitus. Rom J Intern Med 2006; 44: 69-78.

8. Komatsu F, Kagawa Y, Kawabata T, Kaneko Y, Ishiguro K. Relationship of dietary habits and obesity to oxidative stress in Palauan people: compared with Japanese and Mongolian people. Curr Aging Sci 2009; 2: 214-222.

9. Halliwell B. Free radicals, antioxidants and human disease: curiosity, cause or consequence. Lancet 1994; 344: 721-724.

10. Chapple IL, Milward MR, Dietrich T. The prevalence of inflammatory periodontitis is negatively associated with serum antioxidant concentrations. $J$ Nutr 2007; 137: 657-664.

11. Demasi APD, Ceratti D, Furuse C, Cury P, Junqueira JLC, Araújo VC. Expression of peroxiredoxin I in plasma cells of oral inflammatory diseases. Eur J Oral Sci 2007; 115: 334-337.

12. Tamaki N, Tomofuji T, Ekuni D, Yamanaka R, Yamamoto T, Morita M. Short-term effects of non-surgical periodontal treatment on plasma level of reactive oxygen metabolites in patients with chronic periodontitis. J Periodontol 2009; 80: 901-906.

13. Sanbe T, Tomofuji T, Ekuni D, et al. Vitamin $\mathrm{C}$ intake inhibits serum lipid 
peroxidation and osteoclast differentiation on alveolar bone in rats fed on a high-cholesterol diet. Arch Oral Biol 2009; 54: 235-240.

14. Bastard JP, Jardel C, Delattre J, Hainque B, Bruckert E, Oberlin F. Evidence for a link between adipose tissue interleukin-6 content and serum C-reactive protein concentrations in obese subjects. Circulation 1999; 99: 2221-2222.

15. Paraskevas S, Huizinga JD, Loos BG. A systematic review and meta-analyses on C-reactive protein in relation to periodontitis. J Clin Periodontol 2008; 35: 277-290.

16. Devaraj S, Dasu MR, Singh U, Rao LV, Jialal I. C-reactive protein stimulates superoxide anion release and tissue factor activity in vivo. Atherosclerosis 2009; 203: $67-74$.

17. Closhen D, Bender B, Luhmann HJ, Kuhlmann CR. CRP-induced levels of oxidative stress are higher in brain than aortic endothelial cells. Cytokine 2010; 50: 117-120.

18. Bassuk SS, Manson JE. Epidemiological evidence for the role of physical activity in reducing risk of type 2 diabetes and cardiovascular disease. J Appl Physiol 2005; 99: 1193-1204.

19. Gomez-Merino D, Drogou C, Guezennec CY, Chennaoui M. Effects of chronic exercise on cytokine production in white adipose tissue and skeletal muscle of rats. 
Cytokine 2007; 40: 23-29.

20. Lira FS, Rosa JC, Yamashita AS, Koyama CH, Batista ML Jr, Seelaender M. Endurance training induces depot-specific changes in IL-10/TNF-alpha ratio in rat adipose tissue. Cytokine 2009; 45: 80-85.

21. Sakurai T, Izawa T, Kizaki T, et al. Exercise training decreases expression of inflammation-related adipokines through reduction of oxidative stress in rat white adipose tissue. Biochem Biophys Res Commun 2009; 379: 605-609.

22. Dandona P, Mohanty P, Ghanim H, et al. The suppressive effect of dietary restriction and weight loss in the obese on the generation of reactive oxygen species by leukocytes, lipid peroxidation, and protein carbonylation. J Clin Endocrinol Metab 2001; 86: 355-362.

23. Uzun H, Zengin K, Taskin M, Aydin S, Simsek G, Dariyerli N. Changes in leptin, plasminogen activator factor and oxidative stress in morbidly obese patients following open and laparoscopic Swedish adjustable gastric banding. Obes Surg 2004; 14: 659-665.

24. Esposito K, Pontillo A, Di Palo C, Giugliano G, Masella M, Marfella R, Giugliano D. Effect of weight loss and lifestyle changes on vascular inflammatory markers in obese women: a randomized trial. JAMA 2003; 289:1799-1804. 
25. Kasai H. Chemically-based studies on oxidative DNA damage: formation, repair, and mutagenesis. Free Radic Biol Med 2002; 33: 450-456.

26. Valko M, Leibfritz D, Moncol J, Cronin MT, Mazur M, Telser J. Free radicals and antioxidants in normal physiological functions and human disease. Int $J$ Biochem Cell Biol 2007; 39: 44-84.

27. Beverly P. Variation in susceptibility to atherosclerosis among inbred strains of mice. Atherosclerosis 1985; 57: 65-73.

28. Gauthier MS, Couturier K, Charbonneau A, Lavoie JM. Effects of introducing physical training in the course of a 16-week high-fat diet regimen on hepatic steatosis, adipose tissue fat accumulation, and plasma lipid profile. Int J Obes Relat Metab Disord 2004; 28: 1064-1071.

29. Bedford TG, Tipton CM, Wilson NC, Oppliger RA, Gisolfi CV. Maximum oxygen consumption of rats and its changes with various experimental procedures. J Appl Physiol 1979; 47: 1278-1283.

30. Irie K, Tomofuji T, Tamaki N, et al. Effects of ethanol consumption on periodontal inflammation in rats. J Dent Res 2008; 87: 456-460.

31. Lazarevic G, Antic S, Cvetkovic T, Djordjevic V, Vlahovic P, Stefanovic V. Effects of regular exercise on cardiovascular risk factors profile and oxidative stress in 
obese type 2 diabetic patients in regard to SCORE risk. Acta Cardiol 2008; 63: $485-491$

32. Lazarevic G, Antic S, Cvetkovic T, Vlahovic P, Tasic I, Stefanovic V. A physical activity programme and its effects on insulin resistance and oxidative defense in obese male patients with type 2 diabetes mellitus. Diabetes Metab 2006; 32: 583-590.

33. Furukawa S, Fujita T, Shimabukuro M, Iwaki M, Yamada Y, Nakajima Y, Nakayama O, Makishima M, Matsuda M, Shimomura I. Increased oxidative stress in obesity and its impact on metabolic syndrome. J Clin Invest 2004; 114: 1752-1761.

34. Sanbe T, Tomofuji T, Ekuni D, Azuma T, Tamaki N, Yamamoto T. Oral administration of vitamin $\mathrm{C}$ prevents alveolar bone resorption induced by high dietary cholesterol in rats. J Periodontol 2007; 78: 2165-2170.

35. Forsythe LK, Wallace JM, Livingstone MB. Obesity and inflammation: the effects of weight loss. Nutr Res Rev 2008; 21: 117-133.

36. Shih KC, Janckila AJ, Kwok CF, Ho LT, Chou YC, Chao TY. Effects of exercise on insulin sensitivity, inflammatory cytokines, and serum tartrate-resistant acid phosphatase 5a in obese Chinese male adolescents. Metabolism 2010; 59: 144-151. 
Table 1. Body Weight and Fat Pad Weight in Response to 4- and 8-week Interventions $(\mathrm{g}$, Mean $\pm \mathrm{SD})$

\begin{tabular}{lccc}
\hline & RD-Sed & HF-Sed & HF-Tr \\
& $(\mathrm{N}=7)$ & $(\mathrm{N}=7)$ & $(\mathrm{N}=6)$ \\
\hline Body weight at baseline & $319 \pm 9$ & $325 \pm 8$ & $324 \pm 3$ \\
Body weight at 4 weeks & $326 \pm 13$ & $368 \pm 25^{\mathrm{a}}$ & $350 \pm 11$ \\
Body weight at 8 weeks & $434 \pm 32^{*}$ & $476 \pm 17^{\mathrm{a}, *}$ & $429 \pm 27^{\mathrm{b}, *}$ \\
Visceral fat at 4 weeks & $4.0 \pm 0.4$ & $5.5 \pm 1.0^{\mathrm{a}}$ & $5.3 \pm 1.3^{\mathrm{a}}$ \\
Visceral fat at 8 weeks & & & \\
Subcutaneous fat at 4 weeks & $1.6 \pm 0.3$ & $10.5 \pm 1.3^{\mathrm{a}, *}$ & $6.3 \pm 0.8^{\mathrm{a}, \mathrm{b}}$ \\
& & & $2.2 \pm 0.4^{\mathrm{a}}$ \\
Subcutaneous fat at 8 weeks & $3.3 \pm 0.9^{*}$ & $4.5 \pm 0.7^{\mathrm{a}, *}$ & $1.4 \pm 0.4^{\mathrm{a}, \mathrm{b}^{*} *}$
\end{tabular}

${ }^{\text {a }}$ Significantly different from the RD-Sed group, $P<0.05$ (Tukey's method).

${ }^{\mathrm{b}}$ Significantly different from the HF-Sed group, $P<0.05$ (Tukey's method).

* Significantly different from the value at 4 weeks (within groups), $P<0.05$ ( $t$-test). 
Table 2. Histopathological Evaluation in Periodontal Tissues at 4- and 8-week Interventions (Mean \pm SD)

\begin{tabular}{|c|c|c|c|c|c|c|}
\hline & \multicolumn{3}{|c|}{4 Weeks } & \multicolumn{3}{|c|}{8 Weeks } \\
\hline & $\begin{array}{l}\text { RD-Sed } \\
(\mathrm{N}=7)\end{array}$ & $\begin{array}{l}\text { HF-Sed } \\
(\mathrm{N}=7)\end{array}$ & $\begin{array}{l}\mathrm{HF}-\mathrm{Tr} \\
(\mathrm{N}=7)\end{array}$ & $\begin{array}{l}\text { RD-Sed } \\
(\mathrm{N}=7)\end{array}$ & $\begin{array}{l}\text { HF-Sed } \\
(\mathrm{N}=7)\end{array}$ & $\begin{array}{l}\text { HF- } \mathrm{Tr} \\
(\mathrm{N}=6)\end{array}$ \\
\hline $\begin{array}{l}\text { Polymorphonuclear } \\
\text { leukocytes (numbers/ } \\
0.05 \mathrm{~mm} \times 0.1 \mathrm{~mm} \text { ) }\end{array}$ & $0.5 \pm 0.4$ & $0.9 \pm 0.7$ & $1.0 \pm 0.7$ & $0.3 \pm 0.2$ & $0.8 \pm 0.2^{\mathrm{a}}$ & $0.2 \pm 0.2^{\mathrm{b}, *}$ \\
\hline $\begin{array}{l}\text { Distance between the } \\
\text { cemento-enamel } \\
\text { junction and the } \\
\text { alveolar bone crest }(\mu \mathrm{m})\end{array}$ & $569 \pm 94$ & $585 \pm 80$ & $607 \pm 52$ & $574 \pm 157$ & $739 \pm 128^{*}$ & $663 \pm 88$ \\
\hline $\begin{array}{l}\text { TRAP-positive } \\
\text { osteoclasts } \\
\text { (numbers/mm) }\end{array}$ & $2.8 \pm 1.7$ & $3.0 \pm 2.1$ & $3.0 \pm 1.7$ & $2.0 \pm 1.3$ & $7.1 \pm 2.1^{\mathrm{a}, *}$ & $3.0 \pm 1.7^{b}$ \\
\hline
\end{tabular}

${ }^{a}$ Significantly different from the RD-Sed group, $P<0.05$ (Tukey's method).

${ }^{\mathrm{b}}$ Significantly different from the HF-Sed group, $P<0.05$ (Tukey's method).

* Significantly different from the value at 4 weeks (within groups), $P<0.05$ ( $t$-test). 
Table 3. Gingival Oxidative Stress at 4- and 8-week Interventions (Mean \pm SD)

\begin{tabular}{|c|c|c|c|c|c|c|}
\hline & \multicolumn{3}{|c|}{4 Weeks } & \multicolumn{3}{|c|}{8 Weeks } \\
\hline & $\begin{array}{l}\text { RD-Sed } \\
(\mathrm{N}=7)\end{array}$ & $\begin{array}{l}\text { HF-Sed } \\
(\mathrm{N}=7)\end{array}$ & $\begin{array}{l}\text { HF-Tr } \\
(\mathrm{N}=7)\end{array}$ & $\begin{array}{l}\text { RD-Sed } \\
(\mathrm{N}=7)\end{array}$ & $\begin{array}{l}\text { HF-Sed } \\
(\mathrm{N}=7)\end{array}$ & $\begin{array}{l}\text { HF-Tr } \\
(\mathrm{N}=6)\end{array}$ \\
\hline $\begin{array}{l}\text { 8-OHdG level } \\
\text { (ng/mg mtDNA) }\end{array}$ & $0.47 \pm 0.14$ & $1.01 \pm 0.32^{\mathrm{a}}$ & $0.98 \pm 0.49^{\mathrm{a}}$ & $0.55 \pm 0.12$ & $0.97 \pm 0.18^{\mathrm{a}}$ & $0.69 \pm 0.17^{\mathrm{b}}$ \\
\hline GSH/GSSG ratio & $23.2 \pm 1.4$ & $24.0 \pm 1.8$ & $23.7 \pm 1.1$ & $23.6 \pm 5.8$ & $17.0 \pm 3.1^{\mathrm{a}, *}$ & $20.4 \pm 3.7$ \\
\hline
\end{tabular}

${ }^{\text {a }}$ Significantly different from the RD-Sed group, $P<0.05$ (Tukey's method).

${ }^{\mathrm{b}}$ Significantly different from the HF-Sed group, $P<0.05$ (Tukey's method).

* Significantly different from the value at 4 weeks (within groups), $P<0.05$ ( $t$-test). 
Table 4. Serum ROM and CRP Levels at 4- and 8-week Interventions (Mean \pm SD)

\begin{tabular}{|c|c|c|c|c|c|c|}
\hline & \multicolumn{3}{|c|}{4 Weeks } & \multicolumn{3}{|c|}{8 Weeks } \\
\hline & RD-Sed & HF-Sed & HF-Tr & RD-Sed & HF-Sed & HF-Tr \\
\hline & $(\mathrm{N}=7)$ & $(\mathrm{N}=7)$ & $(\mathrm{N}=7)$ & $(\mathrm{N}=7)$ & $(\mathrm{N}=7)$ & $(\mathrm{N}=6)$ \\
\hline ROM (CARR U) & $333 \pm 12$ & $413 \pm 64^{\mathrm{a}}$ & $466 \pm 60^{\mathrm{a}}$ & $407 \pm 19^{*}$ & $1105 \pm 95^{\mathrm{a}, *}$ & $623 \pm 103^{\mathrm{a}, \mathrm{b}, *}$ \\
\hline $\mathrm{CRP}(\mathrm{mg} / \mathrm{mL})$ & $0.40 \pm 0.07$ & $0.52 \pm 0.15^{\mathrm{a}}$ & $0.38 \pm 0.10$ & $0.33 \pm 0.04$ & $0.48 \pm 0.04^{\mathrm{a}}$ & $0.39 \pm 0.02^{\mathrm{b}}$ \\
\hline
\end{tabular}

${ }^{\text {a }}$ Significantly different from the RD-Sed group, $P<0.05$ (Tukey's method).

${ }^{\mathrm{b}}$ Significantly different from the HF-Sed group, $P<0.05$ (Tukey's method).

* Significantly different from the value at 4 weeks (within groups), $P<0.05$ ( $t$-test). 


\section{Figure Legend}

Figure 1. TRAP-positive osteoclasts in rat periodontal ligament at 8 weeks. There were little positive TRAP-positive osteoclasts (arrow, red-stained cytoplasm) in the RD-Sed group (A). The HF-Sed group (B) showed the number of TRAP-positive osteoclasts more than the RD-Sed (A) and the HF-Tr groups $(\mathrm{C})$. AB, alveolar bone and $\mathrm{CM}$, cementum. Scale bar $=30 \mu \mathrm{m}$. 

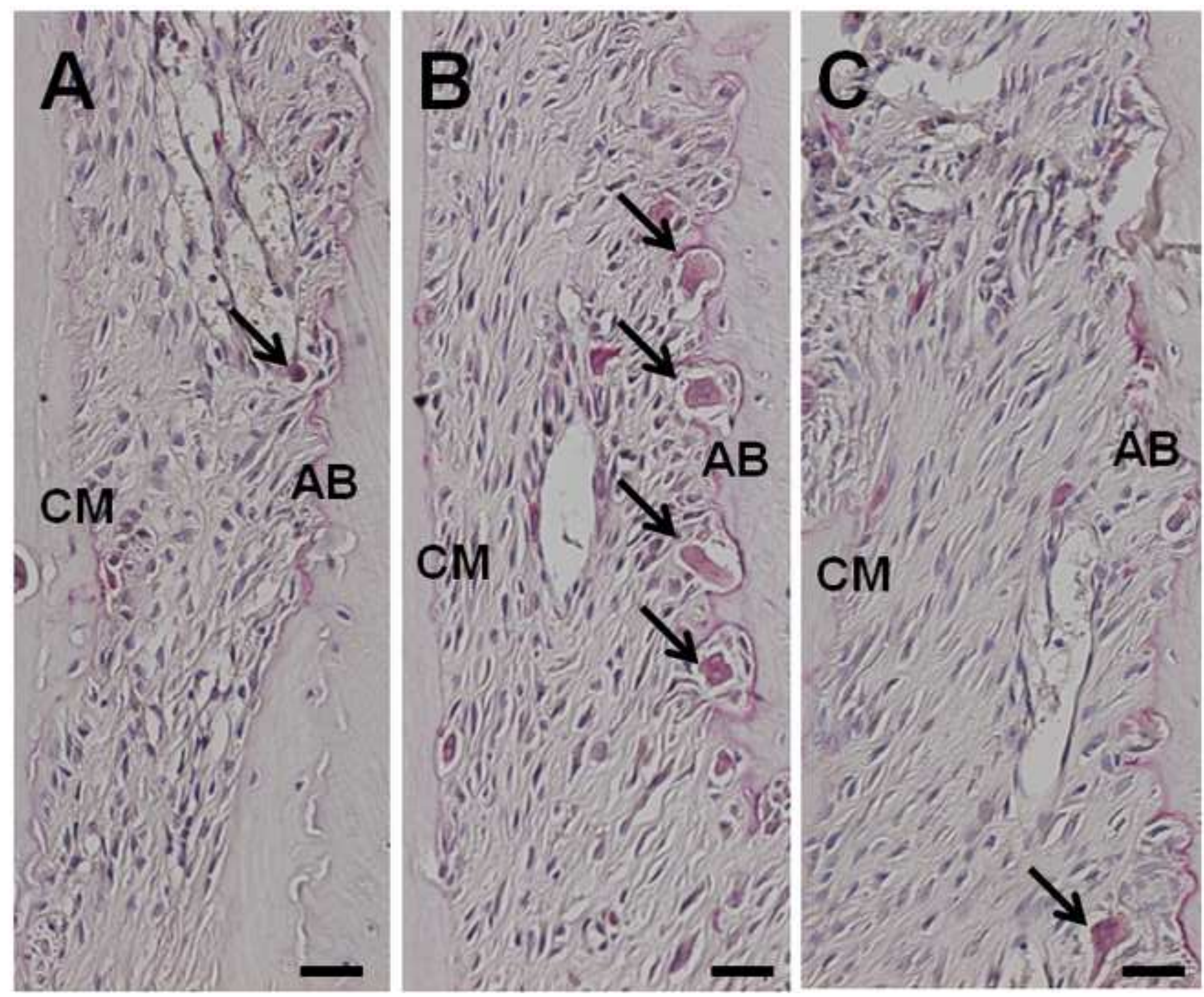